\title{
Group Theory and Quasiprobability Integrals of Wigner Functions
}

\author{
Anthony J. Bracken, ${ }^{\dagger *}$ Demosthenes Ellinas * and James G. Wood $\ddagger$ \\ $\dagger \ddagger C e n t r e$ for Mathematical Physics, Department of Mathematics \\ University of Queensland Brisbane 4072 Australia \\ * Department of Sciences, Section of Mathematics \\ Technical University of Crete GR-731 00 Chania Crete Greece
}

\begin{abstract}
The integral of the Wigner function of a quantum mechanical system over a region or its boundary in the classical phase plane, is called a quasiprobability integral. Unlike a true probability integral, its value may lie outside the interval $[0,1]$. It is characterized by a corresponding selfadjoint operator, to be called a region or contour operator as appropriate, which is determined by the characteristic function of that region or contour. The spectral problem is studied for commuting families of region and contour operators associated with concentric disks and circles of given radius $a$. Their respective eigenvalues are determined as functions of $a$, in terms of the Gauss-Laguerre polynomials. These polynomials provide a basis of vectors in a Hilbert space carrying the positive discrete series representation of the algebra $\mathbf{s} u(1,1) \approx \mathbf{s} o(2,1)$. The explicit relation between the spectra of operators associated with disks and circles with proportional radii, is given in terms of the discrete variable Meixner polynomials.
\end{abstract}

PACS Nos: 03.65.Fd 02.30.Gp 02.20.Sv 42.50.Xa

*ellinas@science.tuc.gr; †ajb@maths.uq.edu.au ; łjgw@maths.uq.edu.au 


\section{Introduction.}

The problem of bounds on integrals of the Wigner quasidistribution function [1] over some subregion or contour of the phase plane for 1-dimensional quantum systems, has been introduced and studied as a theoretical issue [2, 3], with applications in the field of quantum tomography (see e.g. [4, [5]). For a classical (true) probability distribution, such an integral would always have a value in the interval $[0,1]$, but in the quantum case, the upper and lower bounds vary from region to region, and may lie outside the interval $[0,1]$. The relevance of such studies to quantum tomography, is that these bounds define checks on the accuracy of experimental determinations of Wigner functions. It has been shown 2, 3] that, for a given region or contour, the bounds are determined by the maximal and minimal eigenvalues of a corresponding Hermitian operator, to be called the region operator or contour operator, respectively, acting in the space of square integrable (wave)functions of the quantum systems in question. Furthermore, the matrix element of such an operator $K$ between such (wave)functions $\psi$, gives the value of the non-positive-definite quasiprobability integral (QPI), formed by the integral of the Wigner function corresponding to $\psi$, over the region or contour that determines the operator. Attention is therefore focussed on the spectra of various region and contour operators.

In what follows, starting from regions and their boundaries in the phase plane of a classical mechanical system, we use their respective characteristic functions to introduce corresponding region and contour operators. Specifically, we introduce the family of commuting operators $K_{D}(a), K_{C}(a)$, associated with a disk and a circle respectively, with radius $a$, centered at the origin of the phase plane. These operators are diagonal in the Fock space of the number states of the quantum harmonic oscillator. Their spectra, which determine the upper and lower bounds on the corresponding QPI, show a remarkable richness of structure. We show that these spectra, as functions of the radius $a$, are determined by the Gauss-Laguerre (GL) functions [6], acting as basis functions in vector spaces that carry the $d=1 / 2$ positive discrete series representation of the Lie algebra $\mathbf{s o}(2,1) \approx \mathbf{s u}(\mathbf{1}, \mathbf{1})$, involving two different differential operator realizations. Further, for operators $K_{D, C}(\xi a)$ that refer to disks/circles with different radii $\xi a, \xi \in \boldsymbol{R}$, we find their eigenvalues are determined by the GL basis functions of scaled argument $\xi a$, while the latter are expressed as linear combination of the GL basis of argument $a$, with the Meixner discrete polynomials as expansion coefficients.

\section{Quantum Mechanical setting.}

A one-dimensional quantum system is described by means of the HeisenbergWeyl $(H W)$ algebra $\mathbf{h}_{\mathbf{1}}$, with generators $\left\{1, b, b^{\dagger}\right\}$ satisfying the canonical commutation relations $b b^{\dagger}-b^{\dagger} b \equiv\left[b, b^{\dagger}\right]=1,[b, 1]=0=\left[b^{\dagger}, 1\right]$. In addition to the creation operator $b^{\dagger}$, annihilation operator $b$ and unit operator 1 , we also use the number operator $N=b^{\dagger} b$, which satisfies the relations $[N, b]=-b,\left[N, b^{\dagger}\right]=$ $b^{\dagger}$. An irreducible infinite-dimensional representation $\nu$ of $\mathbf{h}_{\mathbf{1}}$ is carried by the 
so-called Fock space $F \cong l_{2}\left(Z_{+}\right)=\overline{\operatorname{span}}\left\{e_{n} \mid n \in Z_{+}\right\}$, with

$$
\begin{aligned}
\nu(b) e_{n} & =\sqrt{n} e_{n-1}, \nu\left(b^{\dagger}\right) e_{n}=\sqrt{n+1} e_{n+1}, \nu(1) e_{n}=e_{n}, \\
\nu(N) e_{n} & =n e_{n}, \nu(\Pi) e_{n}=(-1)^{n} e_{n},
\end{aligned}
$$

where we have also introduced the parity operator $\Pi$ for later use. We adopt the convention to denote an abstract element and its representative by the same symbol. Then, in the representation $\nu, b^{\dagger}$ is the hermitian conjugate of b, i.e. $\langle\psi, b \varphi\rangle_{F}=\left\langle b^{\dagger} \psi, \varphi\right\rangle_{F}$, as a consequence of the orthonormality relations $\left\langle e_{m}, e_{n}\right\rangle_{F}=\delta_{m n}$. Now we introduce the bounded displacement operators

$$
D(\alpha)=\exp \left(\alpha^{*} b-\alpha b^{\dagger}\right): C \longrightarrow B(F) .
$$

Here $\alpha$ is a complex number and $\alpha^{*}$ its complex conjugate. The displacement operators define a projective representation of the abelian group of addition in the complex plane:

$$
\begin{aligned}
D(0) & =1, \quad D(\alpha)^{\dagger}=D(-\alpha), \\
D(\alpha) D(\beta) & =D(\alpha+\beta) e^{i \alpha \times \beta},
\end{aligned}
$$

with $\alpha, \beta \in C \equiv \Gamma$ and $\alpha \times \beta=\operatorname{Re} \alpha \operatorname{Im} \beta-\operatorname{Im} \alpha \operatorname{Re} \beta$. Note that $\Gamma$ is endowed with the geometric structure of a symplectic space and physically is identified with the classical phase space of the quantum mechanical problem dealt with. The family of Wigner operators defined as $\left\{\hat{W}(\alpha)=D(\alpha) \Pi D(\alpha)^{\dagger} \mid \alpha \in \Gamma\right\}$ provides a continously parametrized operator basis for the Hilbert space $\mathcal{S}(F)$ of Hilbert-Schmidt operators on $F$ due to the relation

$$
\langle\hat{W}(\alpha), \hat{W}(\beta)\rangle_{\mathcal{S}} \equiv \operatorname{Tr}\left[\hat{W}(\alpha)^{\dagger} \hat{W}(\beta)\right]=\delta(\alpha-\beta) .
$$

Given $\hat{A}, \hat{B} \in \mathcal{S}(F)$, so that $\langle\hat{A}, \hat{A}\rangle_{\mathcal{S}}<\infty$ etc., then

$$
\hat{A}=\int_{\Gamma}\langle\hat{A}, \hat{W}(\alpha)\rangle_{\mathcal{S}} \hat{W}(\alpha) d^{2} \alpha \equiv \int_{\Gamma} A(\alpha) \hat{W}(\alpha) d^{2} \alpha
$$

and

$$
\langle\hat{A}, \hat{B}\rangle_{\mathcal{S}}=\int_{\Gamma} A(\alpha)^{*} B(\alpha) d^{2} \alpha
$$

where $d^{2} \alpha=d(\operatorname{Re} \alpha) d(\operatorname{Im} \alpha) / \pi$. Of special interest are the the density operators $\rho \in \mathcal{S}(F)$, which comprise the convex set $\mathcal{D}$ of operators satisfying $\rho^{\dagger}=\rho$, $\rho>0$ and $\operatorname{Tr}[\rho]=1$. When expanded in the Wigner operator basis, a density operator furnishes as expansion coefficient, the corresponding Wigner function: $W_{\rho}(\alpha) \equiv\langle\rho, \hat{W}(\alpha)\rangle_{\mathcal{S}}$. Now (7) gives

$$
\operatorname{Tr}(\rho \hat{A})=\int_{\Gamma} A(\alpha) W_{\rho}(\alpha) d^{2} \alpha,
$$

which is interpreted as the expectation value of the observable represented by the operator $\hat{A}$, or function $A(\alpha)$, when the system is in the state described 
by the density operator $\rho$, or by the Wigner function $W_{\rho}$. The set $P$ of 'pure state' density operators have the additional projection operator property, i.e $\rho^{2}=\rho$, and the elements of $P$ are the extremal points of $\mathcal{D}$, i.e. $\mathcal{D}=\operatorname{hull}(P)$. For any $\psi \in F$, we define $\psi(x)=\sum_{n}\left\langle e_{n}, \psi\right\rangle_{F} H_{n}(x) e^{-x^{2} / 2}$, where $H_{n}$ is the (normalized) Hermite polynomial [6], so that

$$
\left\langle\psi_{1}, \psi_{2}\right\rangle_{F}=\int_{R} \psi_{1}(x)^{*} \psi_{2}(x) d x
$$

Now if $\rho \in P$ projects onto $\psi \in F$, the Wigner function corresponding to $\rho$ and $\psi$ takes the form

$$
W_{\psi}(q, p)=\frac{1}{2 \pi} \int_{R} \psi(q+x / 2)^{*} \psi(q-x / 2) e^{i p x} d x
$$

where $q=\operatorname{Re} \alpha, p=\operatorname{Im} \alpha$. The Wigner function was introduced as analogous to a probability density in an exploration of the extent to which quantum mechanics can be cast into the form of a true statistical theory [1, 7, 8]. As is well known and easy to show, $W_{\rho}(q, p)$ corresponding to some density operator $\rho$ is not in general positive everywhere. It is consequently called a quasiprobability function, and some of its previously known bounds, such as $-(1 / \pi) \leq W_{\rho}(q, p) \leq(1 / \pi)$, as well as some recently found ones [2, 3], can be used to help quantify its quasiprobability-density character.

\section{Quasiprobability integrals, region operators and contour operators.}

Let $\chi_{S}(\alpha)$ denote the characteristic function of a given region $S$ in the phase space $\Gamma$, and let $\hat{\chi}_{S}$ denote the corresponding operator given by (6), with $\chi_{S}(\alpha)$ replacing $A(\alpha)$ in the RHS. We refer to $\hat{\chi}_{S}$ as the region operator corresponding to $S$. If $S$ is compact, $\hat{\chi}_{S}$ is bounded, with a discrete spectrum.

Considering (8) in the case $\hat{A}=\hat{\chi}_{S}$, we see that the LHS is the expectation value of $\hat{\chi}_{S}$, in the state $\rho$, while the RHS is the QPI on $S$, namely the integral of $W_{\rho}$ over the support of $\chi_{S}$.

It follows that the QPI on $S$ is bounded above and below by the greatest and least eigenvalues of $\hat{\chi}_{S}$, respectively, and also that these bounds are attained when $\rho$ projects onto the corresponding eigenfunctions of $\hat{\chi}_{S}$.

A region of special interest is the disk of radius $a$, centred on the origin, with region operator the disk operator $\hat{\chi}_{S} \equiv K_{D}(a)$. It is known 2$]$ that $K_{D}(a)$ commutes with $N$ for every $a$. This may be viewed as a consequence of the fact that $N$ generates transformations of $\Gamma$ which leave every such disk invariant. Furthermore, the eigenvalue of $K_{D}(a)$ on the eigenvector $e_{n}$ of $N$ has been found to be given by means of the Laguerre polynomials $L_{n}(x)$ as,

$$
\lambda_{n}^{D}(a)=2(-1)^{n} \int_{0}^{a} L_{n}\left(2 x^{2}\right) e^{-x^{2}} x d x, \quad n=0,1,2, \ldots
$$

Generalising the idea of a region operator, we define 3 for every suitably smooth contour $C$ in $\Gamma$, a contour operator $\hat{\chi}_{C}$ using (6), replacing $A(\alpha)$ in 
the RHS by the generalised characteristic function $\chi_{C}(\alpha)$ of $C$. Here $\chi_{C}(\alpha)$ is defined by the property that, for every smooth function $F(\alpha)$ on $\Gamma$,

$$
\int_{\Gamma} F(\alpha) \chi_{C}(\alpha) d^{2} \alpha=\int_{C} F(\alpha) d l
$$

where $d l$ is the element of length along $C$. We can write

$$
\hat{\chi}_{S}=\int_{S} \hat{W}(\alpha) d^{2} \alpha, \quad \hat{\chi}_{C}=\int_{C} \hat{W}(\alpha) d l .
$$

In the case that $C$ is the circular boundary of the disk of radius $a$, centred on the origin, we can see that the circle operator $\hat{\chi}_{C} \equiv K_{C}(a)$ is given by

$$
K_{C}(a)=\lim _{\epsilon \rightarrow 0} \frac{1}{\epsilon}\left(K_{D}(a+\epsilon)-K_{D}(a)\right) .
$$

Furthermore, it can now be seen that $K_{C}(a)$ also commutes with $N$, and indeed with each $K_{D}(b)$, for every value of $a$ and $b$, and that on $\left\{e_{n}\right\}_{n \in Z_{+}}, K_{C}(a)$ has the eigenvalue

$$
\lambda_{n}^{C}(a)=\frac{d}{d a} \lambda_{n}^{D}(a)=2(-1)^{n} L_{n}\left(2 a^{2}\right) e^{-a^{2}} a, \quad n=0,1,2, \ldots
$$

\section{Results.}

Our main object now is to clarify the relation between the spectra (11) and (15) of commuting concentric circle and disk operators for various radii.

We begin with some group theoretical preliminaries: Let $\mathbf{g}$ denote the Lie algebra $\mathbf{s u}(\mathbf{1}, \mathbf{1})$ with generators $\left\{S_{0}, S_{ \pm}\right\}$in some representation, subject to relations

$$
\left[S_{0}, S_{ \pm}\right]= \pm S_{ \pm},\left[S_{+}, S_{-}\right]=-2 S_{0},
$$

together with $S_{0}^{\dagger}=S_{0}$ and $S_{ \pm}^{\dagger}=S_{\mp}$. The central element $\mathcal{C}=S_{0}^{2}-\frac{1}{2} S_{+} S_{-}+$ $S_{-} S_{+}$belongs to the enveloping algebra $U(\mathbf{s u}(\mathbf{1}, \mathbf{1}))$. Consider in particular the positive discrete series representation $\mathcal{D}_{k}^{+}$labelled by a positive integer or half integer $k$, and carried by a linear space $H$ with formal vectors $\left\{d_{n}^{(k)}\right\}_{n \in Z_{+}}$and actions of the operators 9 ]

$$
S_{ \pm} d_{n}^{(k)}=\mu_{ \pm} d_{n \pm 1}^{(k)}, S_{0} d_{n}^{(k)}=(k+n) d_{n}^{(k)}, \mathcal{C} d_{n}^{(k)}=k(k-1) d_{n}^{(k)},
$$

with $\mu_{-}=\mu_{-}(n)=[n(2 k+n-1)]^{\frac{1}{2}}, \mu_{+}=\mu_{-}(n+1)$. We will consider two different realizations of $\mathcal{D}_{k}^{+}$, called the $\pi$-realization and $\sigma$-realization below. In each, the generators are realized by appropriate differential operators acting on representation spaces isomorphic to $l^{2}\left(Z_{+}\right)$.

$\pi$-Realization. For $M$ real nonzero, the central element and the generators of the realization $\pi: \mathrm{g} \longrightarrow \operatorname{End}\left(H_{\pi}\right)$ of $\mathcal{D}_{k}^{+}$, are denoted $L^{(M)}=\pi(C), L_{0}^{(M)}=$ 
$\pi\left(S_{0}\right), L_{ \pm}^{(M)}=\pi\left(S_{ \pm}\right)$, and act on the Hilbert space $H_{\pi} \approx L_{2}([0, \infty), d r)=$ $\overline{\operatorname{span}}\left\{u_{k, l}^{(M)}(r) \mid M \in R, k \in Z_{+}, l \in\left\{-\frac{1}{2}, 0, \frac{1}{2}, 1, \ldots\right\}, r \in(0, \infty)\right\}$. The basis vectors are 10

$$
u_{k, l}^{(M)}(r)=(-1)^{k}\left(\frac{2 \sqrt{M} k !}{\Gamma\left(k+l+\frac{3}{2}\right)}\right)^{\frac{1}{2}}(\sqrt{M} r)^{l+1} e^{-\frac{M}{2} r^{2}} L_{k}^{l+\frac{1}{2}}\left(M r^{2}\right),
$$

with $L_{a}^{b}(x)$ the associated Laguerre polynomial [6]. In this representation module the generators are realized by differential operators

$$
\begin{aligned}
& L_{0}^{(M)}=\frac{1}{4 M}\left[-\frac{d^{2}}{d r^{2}}+\frac{l(l+1)}{r^{2}}\right]+\frac{M}{4} r^{2}, \\
& L_{ \pm}^{(M)}=-\frac{1}{4 M}\left[-\frac{d^{2}}{d r^{2}}+\frac{l(l+1)}{r^{2}}\right]+\frac{M}{4} r^{2} \mp \frac{1}{2}\left(r \frac{d}{d r}+\frac{1}{2}\right) .
\end{aligned}
$$

Let $d=\frac{1}{2}\left(l+\frac{3}{2}\right)$, then the basis vectors of $H_{\pi}$ are eigenvectors of the central element with eigenvalues determining the representation i.e.

$$
L^{(M)} u_{k, l}^{(M)}(r)=d(d-1) u_{k, l}^{(M)}(r),
$$

while the action of the remaining generators on the basis vectors reads

$$
L_{ \pm}^{(M)} u_{k, l}^{(M)}(r)=\mu_{ \pm} u_{k \pm 1, l}^{(M)}(r), L_{0}^{(M)} u_{k, l}^{(M)}(r)=(d+k) u_{k, l}^{(M)}(r) .
$$

$\sigma$-Realization. Here the central element and the generators of the realization $\sigma: \mathbf{g} \longrightarrow \operatorname{End}\left(H_{\sigma}\right)$ of $\mathcal{D}_{k}^{+}$are denoted $J=\sigma(C), J_{0}=\sigma\left(S_{0}\right), J_{ \pm}=\sigma\left(S_{ \pm}\right)$, and act on the Hilbert space $H_{\sigma} \approx L_{2}\left([0, \infty), r^{w} d r\right)=\overline{\operatorname{span}}\left\{e_{m}^{(k)}(r) \mid k \in R_{+}, m \in\right.$ $\left.Z_{+}, r \in(0, \infty)\right\}$. The basis vectors are [1]

$$
e_{m}^{(k)}(r)=2^{W} \sqrt{\frac{w m !}{\Gamma(2 k+m)}} \exp \left(-r^{w}\right)\left(2 r^{w}\right)^{k-W} L_{m}^{(2 k-1)}\left(2 r^{w}\right),
$$

with $L_{a}^{b}(x)$ the associated Laguerre polynomial and $W=\frac{w+1}{2 w}$ for $w \geq 1$. In this representation module the generators are realized by differential operators

$$
\begin{aligned}
J_{0} & =\frac{1}{2}\left(w^{-2} r^{2-w} p_{r}^{2}+\xi r^{-w}+r^{w}\right), \\
J_{1} & =\frac{1}{2}\left(w^{-2} r^{2-w} p_{r}^{2}-\xi r^{-w}-r^{w}\right), \\
J_{2} & =w^{-1}\left(r p_{r}-\frac{i}{2}(w-1)\right),
\end{aligned}
$$

where $p_{r}=-i(d / d r+1 / r), \xi=k(k-1)-W(W-1)$, and the hermitian generators $J_{1}=\frac{1}{2}\left(J_{+}-J_{-}\right)$and $J_{2}=\frac{1}{2 i}\left(J_{+}-J_{-}\right)$have been introduced.

The basis vectors of $H_{\sigma}$ are eigenvectors of the central element with eigenvalues determining the representation, i.e.

$$
J e_{n}^{(k)}(r)=k(k-1) e_{n}^{(k)}(r),
$$


while the action of the rest of generators on the basis vectors reads

$$
J_{ \pm} e_{n}^{(k)}(r)=\mu_{ \pm} e_{n \pm 1}^{(k)}(r), J_{0} e_{n}^{(k)}(r)=(n+k) e_{n}^{(k)}(r) .
$$

To make connection with the spectrum of the circle operator $K_{C}(a)$ we first observe that for basis elements of $H_{\pi}$ and $H_{\sigma}$, the relation $\langle f(a), g(a)\rangle_{H_{\sigma}}=$ $<a f(a), a g(a)>_{H_{\pi}}$ holds among innner products. The choice of values $w=2$ i.e $W=3 / 4$, leads to the relation $a e_{m}^{\left(\frac{l}{2}+\frac{3}{4}\right)}(a)=u_{(m, l)}^{(2)}(a)$, among the basis vectors. Then we identify the eigenvalues of $K_{C}(a)$ with the basis vectors as follows:

$$
\lambda_{n}^{C}(a)=a \sqrt{a} e_{n}^{\left(\frac{1}{2}\right)}(a)=\sqrt{a} u_{n,-\frac{1}{2}}^{(2)}(a) .
$$

\section{Proposition}

Let $\xi \in R_{+}$, then the spectra $S_{\xi}^{C, D}=\left\{\lambda_{n}^{C, D}(\xi a) \mid a \in R_{+}, n \in Z_{+}\right\}$, and $S_{1}^{C, D}=$ $\left\{\lambda_{n}^{C, D}(a) \mid a \in R_{+}, n \in Z_{+}\right\}$, of the respective circle/disc operators $K_{C, D}(\xi a)$ and $K_{C, D}(a)$, for concentric circles/disks of radii $\xi a$ and a, are related by means of discrete Meixner polynomials $\left\{M_{n}\left(m, 2 k ; c^{2}\right) \mid m, n \in Z_{+}, k=0, \frac{1}{2}, 1, \ldots\right\}$ as follows:

$$
\lambda_{m}^{C, D}(\xi a)=\mathcal{N}_{m} \sum_{n=0}^{\infty} c^{n} M_{n}\left(m, 1 ; c^{2}\right) \lambda_{n}^{C, D}(a)
$$

where the coefficients $\mathcal{N}_{m}, c$ depend on $\xi$.

Proof. We start by first stating the following, independent of any realization of the eigenvalue problem : let $X_{c}=-(c+1 / c) S_{0}+S_{+}+S_{-}, 0<c<1$, and consider $\mathcal{D}_{k}^{(+)}$, the discrete series representation of $\mathbf{g}$ carried by a space with basis elements $\left\{d_{n}^{(k)}\right\}_{n \in Z_{+}}$. Then

$$
X_{c} v_{m}^{(k)}=(c-1 / c)(k+m) v_{m}^{(k)}, \quad m \in N, k \in R_{+},
$$

with

$$
v_{m}^{(k)}=\sum_{n=0}^{\infty} \sqrt{\frac{(2 k)_{n}}{n !}} c^{n} M_{n}\left(m, 2 k ; c^{2}\right) d_{n}^{(k)},
$$

where $(\alpha)_{n}=\Gamma(\alpha+n) / \Gamma(\alpha)$ is the Pochhammer symbol [], and the discrete variable Meixner polynomials are defined in terms of the hypergeometric series [12]:

$$
M_{n}\left(m, 2 k ; c^{2}\right)={ }_{2} F_{1}\left[\begin{array}{c}
-n,-m \\
2 k
\end{array} ; 1-1 / c\right] .
$$

[The history of the spectral theory of operators like $X_{c}$ is rich and interesting in itself, for it illuminates the relation of special functions with the representation theory of $\mathbf{s u}(\mathbf{1}, \mathbf{1})$, and so provides a mathematical framework for our 
group theoretical study of region operators. Our $\pi$-realization is based on work in [10. In [13, the spectral problem of the operator $H=2 J_{0}-J_{+}-J_{-}$in the positive discrete series representation of $\mathbf{s u}(\mathbf{1}, \mathbf{1})$ was shown to be related to generalized Laguerre polynomials. Following this, the analogous problem was addressed [14] for $H=\sigma J_{0}-J_{+}-J_{-},(\sigma \in R)$, and earlier, for a particular representation, in [15. The explicit relation to Meixner polynomials in some other realizations (cf. our $\sigma$-realization) was further studied in [11] for the general operator $H=-(\sigma+1 / c) J_{0}-J_{+}-J_{-},(0<\sigma<1)$.]

Since by comparing (17) and (31) we see that operators $S_{0}$ and $X_{c}$ are isospectral apart from the factor $(c-1 / c)$, so we expect their formal eigenvectors to be related by a unitary transformation. To determine this tranformation we first use the Baker-Champell-Hausdorff formula [16 to rewrite $X_{c}$ in the form

$$
X_{c}=(c-1 / c) e^{i r S_{2}} S_{0} e^{-i r S_{2}},
$$

where $e^{r}=\frac{1+c}{1-c}$. Its action on the eigenvector $v_{m}^{(k)}$ then yields

$$
S_{0} e^{-i r S_{2}} v_{m}^{(k)}=(k+m) e^{-i r S_{2}} v_{m}^{(k)},
$$

which leads to identifying eigenvectors of $S_{0}$ and $X_{c}$ up to the normalization coefficient $\mathcal{N}_{m}$

$$
e^{i r S_{2}} d_{m}^{(k)}=\mathcal{N}_{m} v_{m}^{(k)}=\mathcal{N}_{m} \sum_{n=0}^{\infty}<d_{n}^{(k)}, e^{i r S_{2}} d_{m}^{(k)}>d_{n}^{(k)} .
$$

The right hand side of (36) together with (32) yields

$$
e^{i r S_{2}} d_{m}^{(k)}=\phi_{m} \mathcal{N}_{m} \sum_{n=0}^{\infty} \sqrt{\frac{(2 k)_{n}}{n !}} c^{n} M_{n}\left(m, 2 k ; c^{2}\right) d_{n}^{(k)},
$$

where $\left|\phi_{m}\right|=1$.

We first observe that in order to scale the argument of eigenvalues we need the dilation operator $\exp \left(a \frac{d}{d a}\right)$ and that our choice of $\pi$ and $\sigma$-realizations are such that their respective operators $L_{2}=\frac{1}{2}\left(L_{+}-L_{-}\right)=-\frac{1}{2}\left(a \frac{d}{d a}+\frac{1}{2}\right)$ and $J_{2}=\frac{1}{2 i}\left(J_{+}-J_{-}\right)=\frac{1}{2}\left(a \frac{d}{d a}+\frac{3}{2}\right)$ are linear combination of $a \frac{d}{d a}$, hence $\exp \left(-r L_{2}\right)$ and $\exp \left(i r J_{2}\right)$, acting on a function of $a$, take the respective forms

$$
\exp \left(-r L_{2}\right) f(a)=e^{\frac{r}{4}} f\left(e^{\frac{r}{2}} a\right) \text { and } \exp \left(i r J_{2}\right) f(a)=e^{\frac{3 r}{4}} f\left(e^{\frac{r}{2}} a\right) .
$$

From this equation and the identification (29), we obtain

$$
\exp \left(-r L_{2}+\frac{r}{2}\right) \frac{1}{a} u_{n,-\frac{1}{2}}^{(2)}(a)=\exp \left(i r J_{2}\right) e_{n}^{\left(\frac{1}{2}\right)}(a),
$$

and multiplication of both sides by $a \sqrt{a}$ yields

$$
\lambda_{n}^{C}(\xi a)=\xi a \sqrt{\xi a} e_{n}^{\left(\frac{1}{2}\right)}(\xi a)=\sqrt{\xi a} u_{n,-\frac{1}{2}}^{(2)}(\xi a) .
$$


Finally, by means of (36) and (37) and their respective expressions in $\pi, \sigma$ realizations, and with $\xi=e^{\frac{r}{2}}, c=\frac{e^{r}-1}{e^{r}+1}$ and $\mathcal{N}_{m}=\phi_{m}\left(1-c^{2}\right)^{\frac{1}{2}} c^{m}$, where specifically for the $\pi$-realization, $\phi_{m}=(-1)^{m}$, we obtain

$$
\lambda_{m}^{C}(\xi a)=\mathcal{N}_{m} \sum_{n=0}^{\infty} c^{n} M_{n}\left(m, 1 ; c^{2}\right) \lambda_{n}^{C}(a) .
$$

From this relation and the spectral decomposition of the circle operator in the number state basis, we obtain that

$$
K_{C}\left(e^{\frac{r}{2}} a\right)=\sum_{m=0}^{\infty} \lambda_{m}^{C}\left(e^{\frac{r}{2}} a\right) e_{m} e_{m}^{\dagger}=\sum_{m=0}^{\infty}\left(\mathcal{N}_{m} \sum_{n=0}^{\infty} c^{n} M_{n}\left(m, 1 ; c^{2}\right) \lambda_{n}^{C}(a)\right) e_{m} e_{m}^{\dagger},
$$

or that

$$
K_{C}\left(e^{\frac{r}{2}} a\right)=\exp \left(-r L_{2}+\frac{r}{2}\right) K_{C}(a)=\exp \left(i r J_{2}\right) K_{C}(a) .
$$

In view of the simple relation between circle and disk operator spectra, we are led to integrate the scaled eigenvalues of (41) in order to get analoguous equations for the eigenvalues of the disk operator, namely

$$
\begin{aligned}
\lambda_{m}^{D}(\xi a) & =\int_{0}^{a} \lambda_{m}^{C}(\xi x) d x=\mathcal{N}_{m} \sum_{n=0}^{\infty} c^{n} M_{n}\left(m, 1 ; c^{2}\right) \int_{0}^{a} \lambda_{n}^{C}(x) d x \\
& =\mathcal{N}_{m} \sum_{n=0}^{\infty} c^{n} M_{n}\left(m, 1 ; c^{2}\right) \lambda_{n}^{D}(a)_{\diamond}
\end{aligned}
$$

Below we give a diagrammatic form of the content of the above Proposition: the two ladders of spectra for the disc and circle operators with proportional radii $\lambda_{n}^{C, D}(a)$ and $\lambda_{n}^{C, D}(\xi a)$ respectively, are shown to be related by means of coefficents determined by discrete Meixner polynomials, that are given as matrix elements of the dilation operator in the Gauss-Laguerre basis functions that carry the positive discrete series representation of the algebra $\mathbf{s u}(\mathbf{1}, \mathbf{1}) \approx$ so $(2,1)$. Also in each spectral ladder the step operators of this algebra $J_{ \pm}$or $L_{ \pm}$ operate to create and annihilate eigenvalues of the respective region or contour operators. 


$\begin{array}{lcl}\vdots & \vdots & \vdots \\ J_{-}, L_{-} \downarrow \uparrow J_{+}, L_{+} & & J_{+}, L_{+} \uparrow \downarrow J_{-}, L_{-} \\ \lambda_{2}^{C, D}(\xi a) & \text { Meixner pol. } & \lambda_{2}^{C, D}(a) \\ J_{-}, L_{-} \downarrow \uparrow J_{+}, L_{+} & & J_{+}, L_{+} \uparrow \downarrow J_{-}, L_{-} \\ \lambda_{1}^{C, D}(\xi a) & \stackrel{\text { Meixner pol. }}{\longrightarrow} & \lambda_{1}^{C, D}(a) \\ J_{-}, L_{-} \downarrow \uparrow J_{+}, L_{+} & & J_{+}, L_{+} \uparrow \downarrow J_{-}, L_{-} \\ \lambda_{0}^{C, D}(\xi a) & \stackrel{\text { Meixner pol. }}{\longrightarrow} & \lambda_{0}^{C, D}(a)\end{array}$

\section{Conclusions.}

The spectra of operators associated with integrals of Wigner functions over regions and contours of classical phase space, can have interesting structural properties. These operators form a new type of quantum mechanical observables, with important mathematical and physical meanings. In the case of discs and their boundaries, their eigenvalues, which carry the meaning of the QPI formed by Wigner functions over the corresponding discs and circles, behave as basis functions of a representation space of a Lie algebra of generators. These generators can be used to step up and down the ladder of these eigenvalues. We emphasize this unusual feature: the representation is defined on the spectrum of eigenvalues, not on the underlying function space on which the region or contour operators act.

Because of their intrinsic interest, as well as their potential importance in quantum tomography, it is important to study further the algebraic and functional properties of such operators. A number of open research problems can be mentioned: development of tranformation theory of region and contour operators under maps that induce simple geometric transformations on their associated region or contour of support; temporal evolution equations and behaviour of such operators under Hamiltonian dynamics of their associated quantum systems; clarification of their mathematical relation to covariant phase operators coming from the problem of quantum mechanical angular operators (see e.g [17]); addition rules for region and contour operators associated with many-body quantum systems and their relation to geometric manifestations of quantum entanglement; and finally, the extension of the concept of QPI, region and contour operators, and their spectral problems to phase spaces other than the classical phase plane, such as the sphere and the continuous and discretized torus. We hope to take up some of these problems in future work. 


\section{References}

[1] E.P. Wigner, On the Quantum Correction for Thermodynamic Equilibrium, Phys. Rev. 40 (1932), 749-759.

[2] A.J. Bracken, H.-D. Doebner and J.G. Wood, Bounds on Integrals of the Wigner Function, Phys. Rev. Lett. 83 (1999), 3758-3761.

[3] A.J. Bracken, D. Ellinas and J.G. Wood, Non-Positivity of the Wigner Function and Bounds on Associated Integrals (Proceedings, Wigner Centennial Conference, Pécs, Hungary, 2002, to appear).

[4] U. Leonhardt, Measuring the Quantum State of Light, Cambridge University Press, Cambridge England, 1997.

[5] Quantum State Preparation and Measurement, J. Mod. Opt. 44(1997) 12. Eds. W. P. Schleich and M. G. Raymer.

[6] M. Abramowitz and I.A. Stegun, Handbook of Mathematical Functions (Dover, New York, 1972).

[7] H. Groenewold, On the Principles of Elementary Quantum Mechanics, Physica 12 (1946), 405-460.

[8] J.E. Moyal, Quantum Mechanics as a Statistical Theory, Proc. Camb. Phil. Soc. 45 (1949), 99-124.

[9] N. J. Vilenkin and A. U. Klimyk, Representations of Lie Groups and Special Functions, vol. I, Kluwer Academic Publs. Dordrecht 1991.

[10] R. Floreanini, J. LeTourneux and L. Vinet, Quantum Mechanics and Polynomials of a Discrete Variable, Ann. of Phys. 226 (1993), 331-349.

[11] J. Van der Jeugt and R. Jagannathan, Realizations of $s u(1,1)$ and $U_{q}(s u(1,1))$ and generating functions for orthogonal polynomials, J. Math. Phys. 39 (1998), 5062-5078.

[12] A. F. Nikiforov, S. K. Duslov and V. B. Uvarov, Classical Orthogonal Polynomials of a Discrete Variable, Springer-Verlag, Berlin , 1991.

[13] J. Van der Jeugt, Coupling coefficients for Lie Algebra representations and addition formulas for special functions, J. Math. Phys. 38 (1997),27282740

[14] H. T. Koelink and J. Van der Jeugt, Convolutions for orthogonal polynomials from Lie and quantum algebra representations, SIAM J. Math. Anal. 29 (1998),794-822,

[15] H. Bacry, A unitary representation of $S L(2, R)$, J. Math. Phys. 31(1990), 2061-2077. 
[16] R. Gilmore, Lie Groups and Some of their Applications, (Wiley, New York, 1974).

[17] P. Lahti and J.-P. Pellonpää, Covariant phase observables in Quantum Mechanics, J. Math. Phys. 40 (1999), 4688-4698. 